\title{
A mixed community of actinomycetes produce multiple antibiotics for the fungus farming ant Acromyrmex octospinosus
}

\author{
Jörg Barke ${ }^{1}$, Ryan F Seipke ${ }^{1 \dagger}$, Sabine Grüschow ${ }^{2 \dagger}$, Darren Heavens ${ }^{3}$, Nizar Drou ${ }^{3}$, Mervyn J Bibb ${ }^{4}$, \\ Rebecca JM Goss', Douglas W Yu ${ }^{1,5}$, Matthew I Hutchings ${ }^{1,6^{*}}$
}

\begin{abstract}
Background: Attine ants live in an intensely studied tripartite mutualism with the fungus Leucoagaricus gongylophorus, which provides food to the ants, and with antibiotic-producing actinomycete bacteria. One hypothesis suggests that bacteria from the genus Pseudonocardia are the sole, co-evolved mutualists of attine ants and are transmitted vertically by the queens. A recent study identified a Pseudonocardia-produced antifungal, named dentigerumycin, associated with the lower attine Apterostigma dentigerum consistent with the idea that coevolved Pseudonocardia make novel antibiotics. An alternative possibility is that attine ants sample actinomycete bacteria from the soil, selecting and maintaining those species that make useful antibiotics. Consistent with this idea, a Streptomyces species associated with the higher attine Acromyrmex octospinosus was recently shown to produce the well-known antifungal candicidin. Candicidin production is widespread in environmental isolates of Streptomyces, so this could either be an environmental contaminant or evidence of recruitment of useful actinomycetes from the environment. It should be noted that the two possibilities for actinomycete acquisition are not necessarily mutually exclusive.
\end{abstract}

Results: In order to test these possibilities we isolated bacteria from a geographically distinct population of A. octospinosus and identified a candicidin-producing Streptomyces species, which suggests that they are common mutualists of attine ants, most probably recruited from the environment. We also identified a Pseudonocardia species in the same ant colony that produces an unusual polyene antifungal, providing evidence for co-evolution of Pseudonocardia with A. octospinosus.

Conclusions: Our results show that a combination of co-evolution and environmental sampling results in the diversity of actinomycete symbionts and antibiotics associated with attine ants.

\section{Background}

Fungiculture in the insect world is practised by ants, termites, beetles and gall midges [1]. The best-characterized examples are the attine ants, which are endemic to South and Central America and to the southern USA. The ancestor of these ants evolved the ability to cultivate fungus as a food source around 50 million years ago, leading to the monophyletic tribe Attini, which number 12 genera with more than 230 species. The genera Acromyrmex

\footnotetext{
*Correspondence: m.hutchings@uea.ac.uk

+ Contributed equally

'School of Biological Sciences, University of East Anglia, Norwich, Norwich Research Park, NR4 7TJ, UK

Full list of author information is available at the end of the article
}

and Atta (40 species) evolved 8-12 million years ago and form a branch of the higher attines, also known as leafcutting ants, which are characterized by large colonies of up to several million individuals [2]. Like the other leafcutting ants, the well-studied species Acromyrmex octospinosus forms a mutualism with a single basidiomycete fungus (Agaricales: Lepiotaceae: Leucocoprineae) Leucoagaricus gongylophorus in which they exchange food as well as protection and transport services [3].

The mutualistic fungal garden can be parasitized by a variety of other fungi [4] but the major pathogen of leafcutting ant fungal gardens is a necrotrophic fungus (Ascomycota: anamorphic Hypocreales) in the genus Escovopsis [5]. Around 25\% of the gardens in Panamanian 
ant colonies contain Escovopsis which feed on the fungal cultivar and can destroy fungal gardens, leading to the collapse of the colony [6].

There is evidence that the fungal cultivar produces antibiotics in order to defend itself [7-9] and the ant workers also defend their fungal gardens through a combination of grooming and weeding [8], production of their own antimicrobials through metapleural gland secretions [10] and the application of weedkillers. These weedkillers are natural product antimicrobials produced by symbiotic actinomycete bacteria [7,11-13]. A longstanding theory suggests that bacteria from the genus Pseudonocardia co-evolved with the ants and are transmitted vertically by the gynes (reproductive females) along with the fungal cultivar. However, more recently, evidence has emerged that suggests attine ants are also associated with bacteria from the actinomycete genera Streptomyces and Amycolatopsis and that antibiotic-producing actinomycetes can be horizontally acquired through male dispersal and sampling of actinomycetes from the soil $[7,14]$.

The identities of the antifungals produced by attine ant-associated actinomycetes remain largely unknown. Only two compounds have been identified so far: a previously unknown antifungal named dentigerumycin that is produced by Pseudonocardia species isolated from the lower attines Apterostigma dentigerum and candicidin, a well known antifungal that is produced by Streptomyces species isolated from the higher attine ants belonging to the genus Acromyrmex [12,13]. Pseudonocardia isolated from A. octospinosus also inhibit the growth of Escovopsis in bioassays, but the antifungal compounds have not been isolated or identified [12].

The aims of this work were to isolate and identify actinomycete bacteria from $A$. octospinosus, identify antifungal compounds produced by these bacteria and thereby gain insights into whether the actinomycetes (i) coevolved with the ants, as suggested by unusual antifungal compounds produced by Pseudonocardia mutualists, or (ii) were acquired from the environment, as suggested by the presence of well known antifungals that are widely produced by environmental isolates. We isolated actinomycetes from three colonies of $A$. octospinosus that were collected in Trinidad, identified two Pseudonocardia and nine Streptomyces species and chose single antifungal producing Pseudonocardia and Streptomyces species isolated from the same ant colony for further analysis. The Streptomyces species was found to produce candicidin and is closely related to the candicidin-producing Streptomyces bacteria isolated from A. octospinosus in Panama [12], supporting the hypothesis that candicidin-producing Streptomyces species are common mutualists of higher attines and are probably acquired via environmental sampling. The Pseudonocardia species produces an unusual antifungal compound that is related to the clinically important polyene antifungal nystatin. The isolation of these species suggests that the diversity of actinomycetes associated with attine ants probably occurs through both co-evolution of Pseudonocardia with the ants and environmental sampling.

This work also takes the total number of known antifungals associated with attine ants to three, two of which are associated with $A$. octospinosus, and provides the first direct biochemical evidence that a diversity of actinomycete symbionts translates into a diversity of antifungal compounds in attine ant colonies.

\section{Results}

Isolation and bioassay of actinomycetes

A. octospinosus ants from three colonies collected in Trinidad were either streaked directly onto HC and MS agar plates or washed in sterile water which was then spread onto the agar. Actinomycete colonies were purified by restreaking and then examined by light microscopy and identified by $16 \mathrm{~S}$ rDNA sequencing. Together with bacteria from other genera (Tsukamurella and Nocardiopsis) two Pseudonocardia (P1-P2) and nine Streptomyces (S1-S9) strains were isolated and identified (Figure 1, GenBank accession HM179225-HM179235). All bacterial strains were screened in bioassays against a strain of Escovopsis weberi isolated from an A. octospinosus nest and against Candida albicans, a human pathogen. Bioassays revealed that strains P1, S3, S4, S5 and S9 inhibit the growth of $E$. weberi when grown on MS agar (Figure 2) while P1, S3, S4 and S5 also inhibit the growth of C. albicans (Figure 3). The Pseudonocardia P1 strain has weak activity against $E$. weberi and very weak activity against $C$. albicans (Figures 2 and 3 ).

\section{Streptomyces S4 makes candicidin}

A previous study revealed that a Streptomyces strain isolated from A. octospinosus in Panama makes the polyene antifungal candicidin [12] and a polymerase chain reaction (PCR) analysis of the nine Streptomyces and two Pseudonocardia strains using primers used by Haeder et al. in their study revealed that only Streptomyces S4 and S5 contain the candicidin biosynthesis genes $f s c M$ and $f s c P$ (Additional Files 1 and 2). Candicidin production was confirmed using liquid chromatography (LC) followed by tandem mass spectrometry (MS/MS) on butanol-extracted culture supernatants of Streptomyces S4 (Additional File 3). The $f s c M$ and $f s c P$ genes were not found in P1, S3, or S9, which suggests that they are producing antifungals not previously identified in the A. octospinosus mutualism. The PCR product amplified using $f s c P$ primers in the $S 9$ sample was sequenced and is not $f_{s c P}$, consistent with its slightly larger size (Additional File 1). 

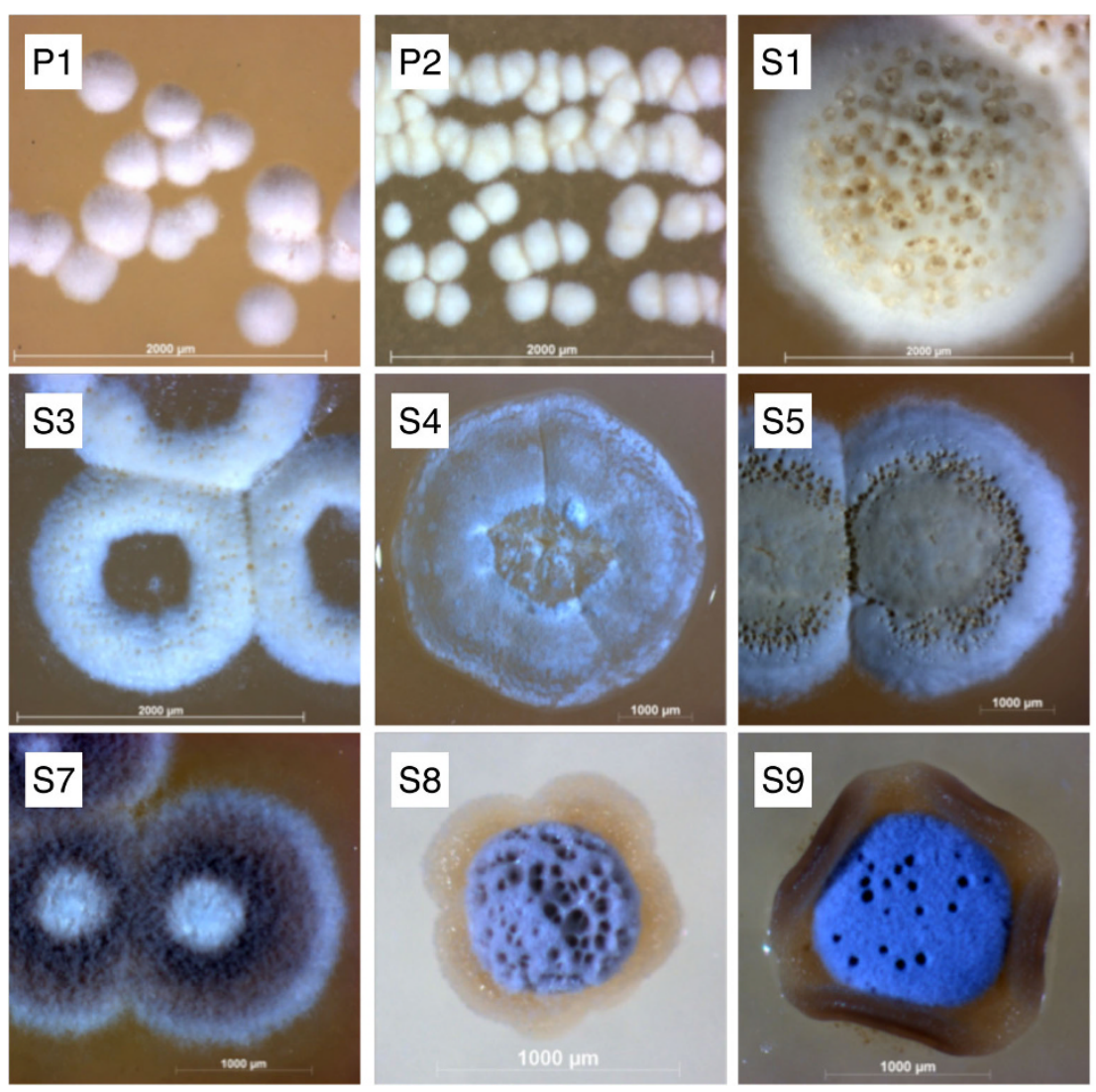
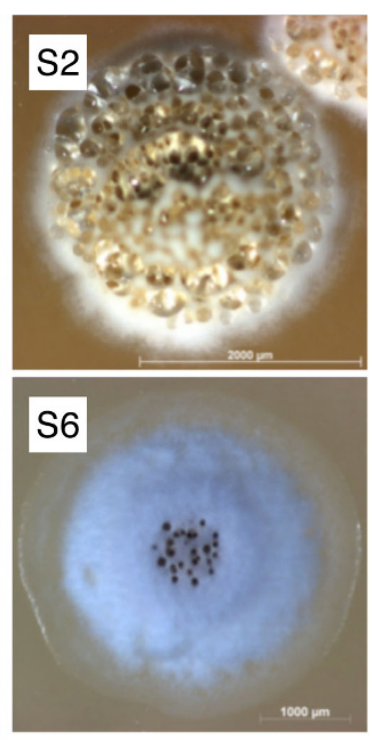

Figure 1 Actinomycete species isolated from attine ants. Actinomycete species isolated from Acromyrmex octospinosus worker ants viewed under a light microscope at $40 \times$ magnification. Streptomyces strains are numbered S1-S9 and Pseudonocardia strains P1-P2.

\section{Genome scanning of Pseudonocardia P1}

Pseudonocardia P1, isolated from the same ant colony as Streptomyces S4, produces a relatively small zone of inhibition in bioassays against $E$. weberi and a very small zone of inhibition against C. albicans (Figures 2 and 3). Furthermore, the antifungal activity of Pseudonocardia P1 was only detected on solid growth medium. This combination of factors made it difficult to purify sufficient antifungal compound(s) for analysis and identification. In order to gain further insight into the antifungal (s) produced by Pseudonocardia P1, we used 454-pyrosequencing to scan the genome of strain P1 (GenBank accession ADUJ00000000; Additional File 4). Analysis of the annotated contigs from this sequencing project revealed several polyketide synthase (PKS) gene fragments with $>90 \%$ amino acid identity to proteins involved in the biosynthesis of an antifungal compound named nystatin-like Pseudonocardia polyene (NPP) that is produced by Pseudonocardia autotrophica [15]. NPP is related to nystatin, a polyene antifungal that is made by Streptomyces noursei $[16,17]$.

In order to determine whether or not Pseudonocardia P1 contains the entire biosynthetic gene cluster for a nystatin-like compound, contigs were aligned against the characterized NPP biosynthetic gene cluster from P. autotrophica (see Methods and Additional File 5). The tiled contigs spanned the entire cluster, including the six PKS genes that assemble the nystatin aglycone, the non-sugar containing backbone of nystatin. Fulllength coding sequences were captured for 11 genes (nypF, nypH, nypDIII, nypL, nypN, nypDII, nypDI, nypE, $n y p O, n y p R I V, n y p M)$ that are proposed to be primarily involved in the post PKS-modification of the nystatin aglycone and two new genes, nyp $Y$ and nypZ, with unknown functions (Table 1) [16]. Interestingly, a second glycosyltransferase, absent in $S$. noursei and $P$. autotrophica, is present in the nyp gene cluster and we have named it nypY (Table 1). The NypY protein belongs to the same glycosyltransferase family as NypDI, however it displays only $42 \%$ amino acid identity to NypDI and is therefore unlikely to be a functionally redundant copy of NypDI. This genome analysis strongly suggested that Pseudonocardia P1 has the genetic capacity to produce a nystatin-like polyene antifungal. PCR screening of the Pseudonocardia P2 strain and the nine Streptomyces strains isolated in this study 

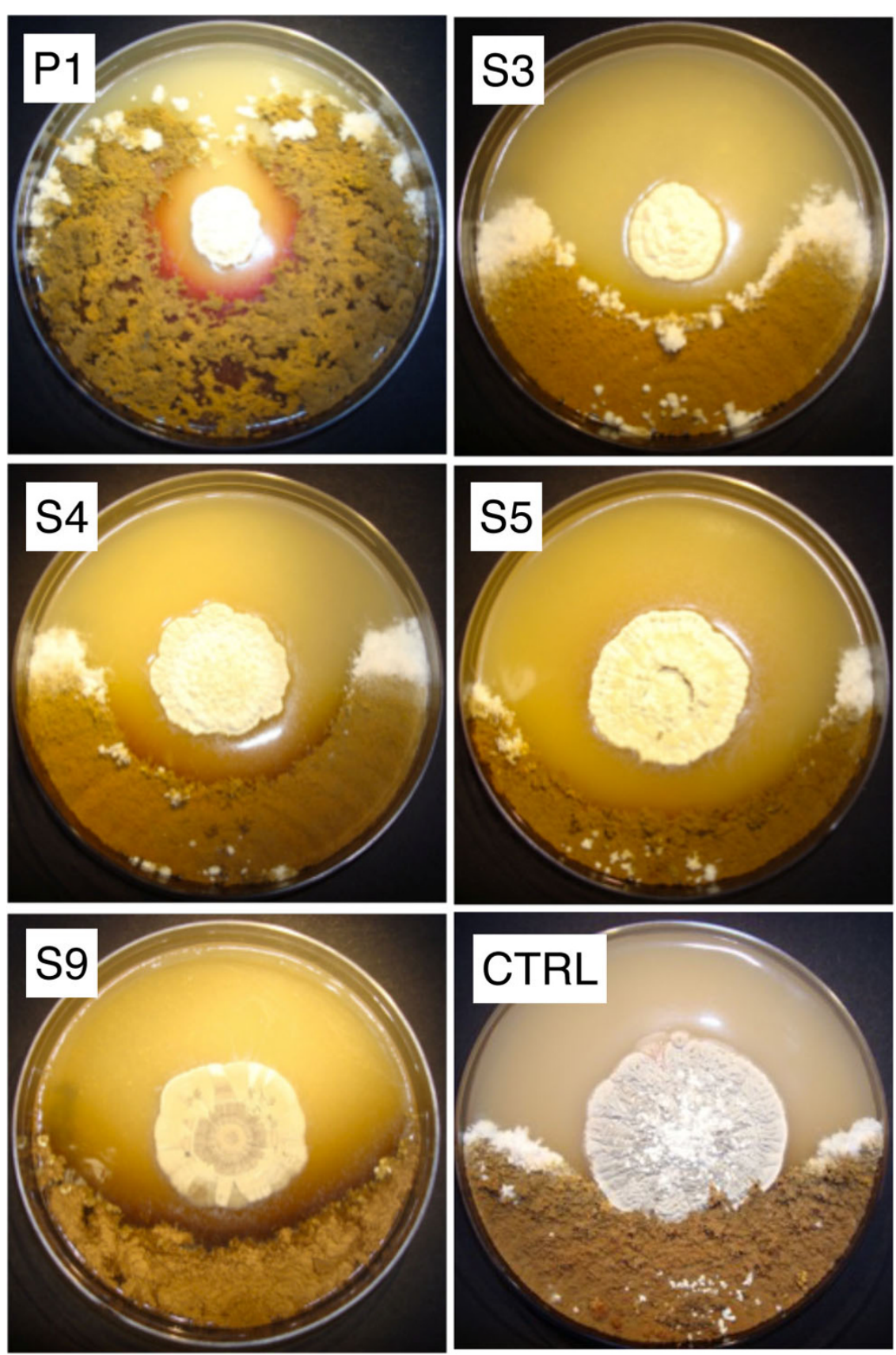

Figure 2 Antifungal bioassays against Escovopsis. Bioassays against the fungal garden parasite Escovopsis weberi. The actinomycete strains S3, S4, S5, S9 and P1 formed clear inhibition zones while the control strain, Streptomyes lividans, produced no zone of inhibition and was overgrown by the nest parasite.

suggests that none of them contain biosynthetic genes for a nystatin-like antifungal (Additional File 2).

\section{Identification of a nystatin-like compound in Pseudonocardia P1}

In order to determine whether Pseudonocardia P1 produces a nystatin-like antifungal compound, extracts of Pseudonocardia P1 were analysed by LC-MS/MS and compared to a nystatin $\mathrm{A}_{1}$ standard (Figure 4). Molecular ions for nystatin $A_{1}(m / z 926.5)$ or for NPP $(m / z$ 1129.6), produced by $P$. autotrophica [15] were not detected. However, a compound with a similar retention time on high-performance liquid chromatography (HPLC) to nystatin $\mathrm{A}_{1}$ and with a molecular ion of $\mathrm{m} / \mathrm{z}$ 1088.6 was identified (Figure 4a and b). This compound clearly, though somewhat concealed by the absorption 

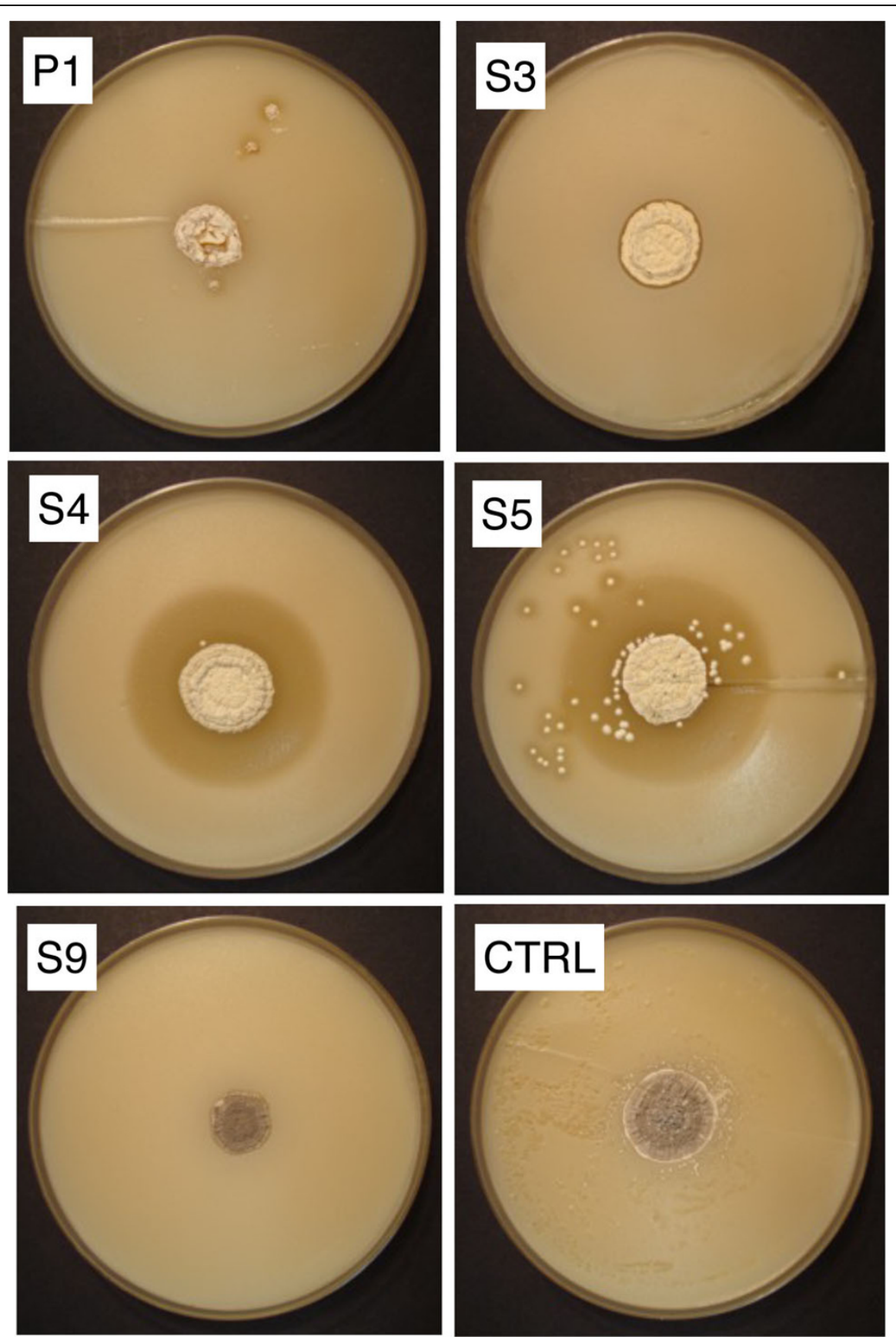

Figure 3 Antifungal bioassays against Candida. Bioassays against the human pathogen Candida albicans. S4, S5 and, to a lesser extent, P1 all inhibit the growth of C. albicans whereas the control strain Streptomyes lividans is overgrown.

of co-eluting peaks, shows the characteristic polyene absorption bands in its ultraviolet spectrum (absorption maxima at 292, 305 and $320 \mathrm{~nm}$, Figure 4e). Together with the presence of nystatin-like biosynthetic genes in Pseudonocardia P1, the LC-MS/MS results strongly suggested that the P1-derived extract contained a nystatinlike compound. We have tentatively named this compound nystatin P1.
The mass difference of 162 observed between nystatin P1 and nystatin $A_{1}$ suggested that nystatin P1 contains an additional hexose molecule. MS/MS fragmentation of the nystatin P1 ion $(m / z$ 1088.6) resulted in a series of product ions that are very similar to those derived from nystatin $\mathrm{A}_{1}$ (Figure 4c). All of the fragment ions corresponding to the nystatin P1 aglycone have corresponding counterparts in the nystatin $A_{1}$ standard (Figure $4 d$ ). 
Table 1 Nystatin P1 biosynthetic genes

\begin{tabular}{llllc}
\hline Contig ID & Pseudonocardia sp. P1 protein & Proposed function* & P. autotrophica ortholog & Identity (\%) \\
\hline PP100949 & NypF & Phosphopantetheinyl transferase & NppF & 89 \\
PP100949 & NypY & Glycosyltransferase & Nonet & - \\
PP100949 & NypZ & Metallophosphoesterase & Noneł & NppH \\
PP100398 & NypH & ABC transporter & NppDIII & 85 \\
PP100398 & NypDIII & dGDP-mannose-4,6-dehydratase & NppL & 96 \\
PP100400 & NypL & P450 monooxygenase & NppN & 84 \\
PP100400 & NypN & P450 monooxygenase & NppDII & 94 \\
PP100400 & NypDII & Aminotransferase & NppDI & 96 \\
PP100400 & NypDI & Glycosyltransferase & NppE & 92 \\
PP100821 & NypE & Thioesterase & NppO & 92 \\
PP100306 & NypO & Acyl-CoA decarboxylase & NppRIV \\
PP100306 & NypRIV & LuxR transcriptional regulator & NppM§ \\
PP100306 & NypM & Hypothetical protein & 96 \\
\hline
\end{tabular}

*Proposed function of full length nystatin P1 biosynthetic (nyp) genes present in the draft genome of Pseudonocardia sp. P1 (Genbank accession ADUJ00000000). † NypY is a glycosyltransferase unique to the nystatin P1 biosynthetic gene cluster and is not orthologous to proteins in the nystatin-like Pseudonocardia polyene (NPP) biosynthetic gene cluster from P. autotrophica (AC $=$ EU108007) or the nystatin biosynthetic gene cluster from Streptomyces nouresi (AC $=$ AF263912).

₹ The nystatin P1 and NPP biosynthetic gene clusters contain a putative metallophosphoesterase downstream of nypH and nppH, respectively that is not present in the nystatin biosynthetic gene cluster from S. nouresi. This open reading frame was not originally annotated by Kim et al. [15] and we have therefore given the Pseudonocardia P1 ortholog the name of nypZ.

$\S$ nypM encodes a hypothetical protein with high homology to NppM, which is annotated as a putative ferredoxin [15], however amino acid homology-based database searches failed to reveal homology to ferredoxin proteins.

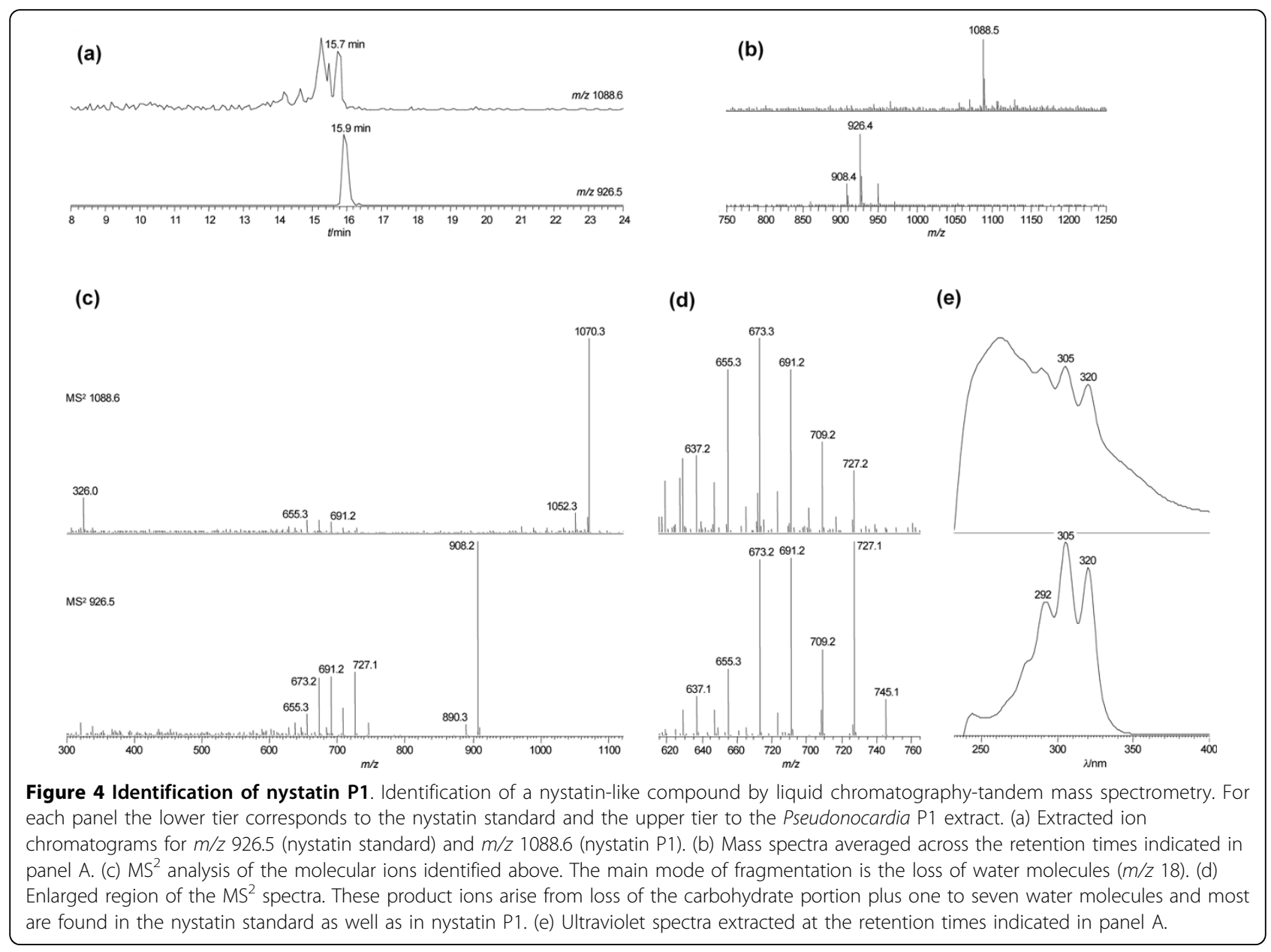


These data strongly suggested that the aglycone (backbone) of nystatin $\mathrm{A}_{1}$ and nystatin $\mathrm{P} 1$ is the same. Interestingly, the product ion with $m / z 326$ is consistent with a mycosamine-hexose disaccharide and was only observed for nystatin P1. Further fragmentation of the $\mathrm{m} / \mathrm{z} 326$ ion species corroborated the disaccharide nature of this moiety (Additional File 6).

The exact identity of the sugar molecules is, of course, speculative. Mycosamine is a probable component of nystatin P1 because this aminosugar is found in nystatin $A_{1}$ and all the necessary genes for its biosynthesis and attachment to the aglycone have been identified in Pseudonocardia P1 (Table 1). Glucose is frequently found as a substituent in bacterial natural products. However, other natural hexoses such as mannose or galactose are also good candidates for the second sugar substituent. The attachment of the hexose to give nystatin P1 is most likely to be executed by the glycosyltransferase NypY (see above). The presence of the disaccharide in MS/MS furthermore suggested that the nystatin P1 aglycone is substituted at one position with a mycosaminehexose moiety rather than the two sugar molecules being attached at separate positions.

\section{Discussion}

We isolated actinomycetes from $A$. octospinosus garden worker ants and, in a single colony of ants, identified a Pseudonocardia and a Streptomyces species that produce antifungals in laboratory culture. The Streptomyces species, which we named S4, contains candicidin biosynthesis genes (Additional Files 1 and 2) and produces candicidin (Additional File 3), consistent with a report on antifungal-producing actinomycetes associated with A. octospinosus [12]. The actinomycetes studied in this work were isolated from $A$. octospinosus ants collected in Trinidad, whereas the previous study used $A$. octospinosus ants collected in Panama [12]. However, despite this geographic separation, the candicidin-producing Streptomyces strains identified in the two studies show 99\% $16 \mathrm{~S}$ rDNA sequence identity suggesting that candicidin-producing Streptomyces are common mutualists of A. octospinosus. Candicidin-producing Streptomyces are widespread in the environment [18] and attine ants most likely acquire them selectively from the soil.

The Pseudonocardia species P1, isolated from the same colony as Streptomyces S4, showed relatively weak antifungal activity that was only observed in cultures grown on solid growth medium. This made it difficult to purify enough of the compound for analysis and identification. Using a genome scanning approach we identified a biosynthetic gene cluster for a polyene antifungal in Pseudonocardia P1 and then isolated and identified this antifungal using LC-MS/MS. This combined chemical and genomic approach provides a powerful tool for identifying and isolating new antibiotics and confirmed that Pseudonocardia P1 produces a polyene antifungal that we have tentatively named nystatin P1. This compound is markedly different from the antifungal dentigerumycin produced by Pseudonocardia associated with the lower attine ant species $A$. dentigerum [13] although it is notable that both Pseudonocardia strains are making previously unknown antifungals, consistent with the idea that the Pseudonocardia mutualists co-evolved with attine ants. We did not detect any compounds in extracts from Pseudonocardia P1 agar plates and mycelium that matched the isotopic mass of dentigerumycin. However, since the biosynthetic gene cluster for this compound is not known, we cannot exclude the possibility that this strain also has the ability to make dentigerumycin.

Taken together, this work provides the first direct evidence that individual leaf-cutting ant colonies have access to multiple antifungals via the diversity of hosted actinomycetes and increases the number of known antifungals used by attine ants to three. This work also provides evidence to support the two current possibilities for the identity and acquisition of mutualistic bacteria, Pseudonocardia co-evolution, and the environmental acquisition of useful actinomycetes. This strongly suggests that both possibilities apply, at least in the attine species A. octospinosus. Careful experimental work will be needed in order to demonstrate that multiple compounds are in fact produced and confer benefits in vivo [19]. It is interesting that the only two antifungal compounds to be isolated and identified from A. octospinosus colonies so far are polyenes, which are active against dimorphic fungi, yeasts (Candida) and molds (Escovopsis), but which apparently do not kill the fungal cultivar [12]. The isolation of a nystatin-like polyene from a leafcutting ant-associated Pseudonocardia species in this work agrees with the report by Sen et al. [11] that some Pseudonocardia bacteria associated with attine ants have non-specific antibiotic properties that inhibit a range of fungi and are not targeted specifically at Escovopsis [11].

The advantage to the ants of deploying two antifungals is not clear. Polyene antifungals are thought to work by interacting hydrophobically with ergosterol in the fungal cell membrane and forming channels that increase membrane permeability [20], but this may not be their only mechanism of action [21], and there may therefore be some advantage to the ants in using more than one. However, as fungi do not develop resistance to polyene antifungals (at least in a clinical setting), it is unlikely that resistance is the basis for any such advantage. Nevertheless, as candicidin and nystatin are not antibacterial, neither of these compounds is likely to be involved in competition amongst the bacteria for host resources. Thus, the identities of these two antifungal 
compounds are consistent with the longstanding hypothesis that these actinomycete associates of leafcutting ants can be mutualists of the ant and the attine fungus, provided that the compounds are applied correctly by the ant [11].

\section{Conclusions}

We used a combined genomic and chemical approach that has proven useful for the identification of a new antifungal associated with Acromyrmex ants, this time produced by their Pseudonocardia mutualist. This approach should stimulate further chemical ecology studies of insect fungiculture systems, which are widespread in nature and which are likely to use symbiotic antibiotic-producing bacteria to protect their fungal partners [1]. We also provide evidence that supports both of the possibilities proposed to explain the mutualism between actinomycetes and attine ants-co-evolution of Pseudonocardia with attine ants and environmental sampling by the ants of useful antibiotic-producing bacteria. We propose that these possibilities are not mutually exclusive and that both are likely to apply to both attine ants and other systems of insect fungiculture.

\section{Methods}

\section{Bacterial isolation and identification}

Ants from three $A$. octospinosus colonies collected in Trinidad and Tobago were streaked onto hydrolysed chitin (HC) and mannitol plus soya flour (MS) agar plates $[22,23]$ containing the antifungals nystatin and cycloheximide at final concentrations of $0.05 \mathrm{mg} / \mathrm{mL}$. The remainder of the ants were washed in sterile water which was then spread onto HC and MS agar plates. Actinomycete isolates were colony purified and stored in $20 \%$ glycerol at $-20^{\circ} \mathrm{C}$. Genomic DNA was isolated from actinomycetes as described [23].

\section{$16 \mathrm{~S}$ rDNA analysis}

A 1000 bp fragment of the $16 \mathrm{~S}$ ribosomal DNA gene was PCR-amplified using the following primers: 533F 5'GTGCCAGCMGCCGCGGTAA-3' [24] and 1492R 5'GGTTACCTTGTTACGACTT-3' [25]. The resulting PCR products were gel purified, sequenced (The Genome Analysis Centre, http://www.tgac.bbsrc.ac.uk/) and subsequently used to query the Green Genes database http://greengenes.lbl.gov/cgi-bin/nph-simrank_interface. cgi.

\section{Bioassays against Escovopsis and Candida}

Spores $(50 \mu \mathrm{L})$ of each actinomycete were inoculated into $10 \mathrm{~mL}$ liquid TSB/YEME (1:1) [23] and grown on a shaker $\left(260 \mathrm{rpm}, 30^{\circ} \mathrm{C}\right)$ for three days in order to generate mycelium. The mycelium was collected by centrifugation and resuspended in fresh TSB/YEME to yield a concentrated cell paste. The centre of an MS plate was inoculated with either $10 \mu \mathrm{L}$ sterile TSB/ YEME (negative control) or $10 \mu \mathrm{L}$ of the concentrated cell paste and incubated for 10 days at $22^{\circ} \mathrm{C}$, at which point the edge of the plate was inoculated with a small amount of mycelium of Escovopsis weberi (CBS 110660). The Escovopsis strain used in this study was obtained from CBS Fungal Biodiversity Centre http://www.cbs. knaw.nl and maintained on MS agar containing carbenicillin and streptomycin each at final concentrations of $0.05 \mathrm{mg} / \mathrm{mL}$. Alternatively, C. albicans was inoculated into soft $(0.5 \%)$ Lysogeny Broth agar, which was then was used to overlay the plate containing the actinomycete.

\section{4-pyrosequencing and analysis}

Genomic DNA was quantified using the Quant-it dsDNA HS Assay Kit (Invitrogen, CA, USA) and measured on a Qubit fluorometer (Invitrogen). An aliquot of $5 \mu \mathrm{g}$ was used to generate the single stranded library for 454 pyrosequencing using the GS Titanium General Library Prep Kit according to the manufacturer's protocol (Roche, Hertfordshire, UK) except that, rather than fragmenting by nebulization, DNA was fragmented in a $100 \mu \mathrm{L}$ volume using the Covaris-S2 ultra sonicator (K Biosciences, PA, USA) with the following settings-Mode: Frequency Sweep, Duty Cycle: 5\%, Intensity: 3, Cycle Burst: 200 for two continuous cycles of 45 s. Library quality and quantity was assessed by running $1 \mu \mathrm{L}$ of the library on a RNA PICO 6000 labchip (Agilent, CA, USA) and an emPCR titration was used to determine the optimal number of molecules per bead required to achieve the targeted $8 \%$ enrichment for the full scale emPCR. Approximately 790,000 enriched templated beads were subjected to 454 pyrosequencing on a quarter of a picotitre plate on the GS FLX sequencer (Roche) using the GS FLX Titanium Chemistry. The sequence reads were quality filtered and assembled into contigs using the Newbler Assembly v2 software (Roche).

Contigs were annotated using the Rapid Annotation Seed Technology Server [26]. Coding sequences annotated as polyketide synthases were extracted and inspected further by BlastP analysis against the National Center for Biotechnology Information non-redundant protein database, as well as Pfam [27] and non-ribosomal peptide synthetases-PKS [28]. NUCmer [29] using an $80 \%$ cutoff and the show-tiling utility were used to tile contigs to the Pseudonocardia autotrophica biosynthetic gene cluster for NPP [15]. Microsoft Excel was used to convert the output of the NUCmer show-tiling utility to Gene Finder Format and visualized using Artemis (release 11.22) [30]. 


\section{LC-MS analysis}

The residue obtained from butanol-extracted Streptomyces S4 cultures $(50 \mathrm{~mL})$ grown in liquid MS was redissolved in $50 \%$ aqueous methanol $(0.3 \mathrm{~mL})$. The samples were centrifuged at maximum speed prior to injection $(5 \mu \mathrm{L})$ into a Shimadzu single quadrupole LCMS-2010A mass spectrometer equipped with Prominence HPLC system. Compounds were separated on a Waters XBridge ${ }^{\mathrm{m}} \mathrm{C} 183.5 \mu \mathrm{m} 2.1 \times 100 \mathrm{~mm}$ column using the following gradient (solvent A: 0.1 formic acid in water, solvent B: $0.1 \%$ formic acid in acetonitrile, flow rate $0.35 \mathrm{~mL} \mathrm{~min}^{-1}$ ): $0.01-0.5 \mathrm{~min} 15 \% \mathrm{~B}, 0.5-14 \mathrm{~min} 15-$ 95\%B, 14-16 min 95\%B, 16-16.5 min 95-15\%B, 16.5-19 min $15 \% \mathrm{~B}$. Mass spectra were acquired in positive ion mode with the capillary voltage set to $1.3 \mathrm{kV}$.

A sporulating culture of the Pseudonocardia P1 isolate on MS agar was extracted twice with methanol (200 $\mathrm{mL}$ ). The solvent was removed under reduced pressure and the residue redissolved in $50 \%$ aqueous methanol $(150 \mu \mathrm{L})$. An authentic nystatin $\mathrm{A}_{1}$ standard (SigmaAldrich, MO, USA) was prepared at $0.1 \mathrm{mg} \mathrm{mL}^{-1}$ in $50 \%$ aqueous methanol. Immediately before LC-MS analysis, the crude extract and the standard were diluted twofold with $20 \%$ aqueous methanol and spun in a microcentrifuge at maximum speed for 4 min to remove any insoluble matter. Only the supernatant was used for injection $(5 \mu \mathrm{L})$. The samples were run on a Surveyor HPLC system attached to a LCQ DecaXPplus ion trap mass spectrometer (both Thermo Fisher, MA, USA). Separation was on a $100 \times 2 \mathrm{~mm} 3 \mu$ Luna C18(2) column (Phenomenex) with $0.1 \%$ formic acid in water as solvent $\mathrm{A}$ and methanol as solvent $\mathrm{B}$ using the following gradient: 0-20 min $20-95 \%$ B, 20-22 min 95\% B, 22-23 min 95$20 \% \mathrm{~B}, 23-30 \mathrm{~min} 20 \% \mathrm{~B}$. The flow rate was set to 260 $\mu \mathrm{L} \min ^{-1}$ and the column temperature was maintained at $30^{\circ} \mathrm{C}$. Detection was by ultraviolet (full spectra from 200-600 nm) and by positive electrospray MS using spray chamber conditions of $350^{\circ} \mathrm{C}$ capillary temperature, 50 units sheath gas, five units auxiliary gas, and 5.2 $\mathrm{kV}$ spray voltage. Targeted $\mathrm{MS}^{2}$ with $\mathrm{S} 4$ and P1 extracts was performed with $35 \%$ collision energy and an isolation width of $m / z 4.0$.

\section{Additional material}

Additional file 1: Detecting candicidin biosynthesis genes using polymerase chain reaction (PCR). PCR analysis of antifungal producers using primers against candicin biosynthesis genes $\mathbf{f s} \boldsymbol{C M}$ and $\boldsymbol{f s c} \boldsymbol{P}$. Sequence identities to Haeder et al. [12]: $\mathbf{f s c} \boldsymbol{M}$ gene, $\mathbf{S 4}=100 \%, \mathrm{~S} 5=$ 99\%; $\boldsymbol{f s c} \boldsymbol{P}$ gene: $\boldsymbol{S} 4=98 \%$ and $\mathrm{S} 5=98 \%$
Additional file 2: Streptomyces and Pseudonocardia strains identified in this study. The Pseudonocardia and Streptomyces strains isolated in this study are listed with the Acromyrmex octospinosus colony they were isolated from $(1,2$ or 3$)$, the accession numbers for their $16 \mathrm{~S}$ ribosomal DNA (rDNA) sequences, the top National Center for Biotechnology Information Blast hits for each of their $16 \mathrm{~S}$ rDNA sequences and the percentage identity to these BLAST hits. Also noted are the results from polymerase chain reaction testing for the candicidin biosynthetic genes $f_{S S M} \mathrm{Cnd}{ }_{\mathrm{SSC}} \mathrm{P}$ using primers from a previous study [12] and the nystatinlike Pseudonocardia polyene biosynthetic gene nppDIII using the primer set RFS84 (CAGATCCGCTTCTACCAGG) and RFS85

(CGCACCGAGTGCATCTG).

Additional file 3: Liquid chromatography-tandem mass spectrometry (LC-MS/MS) identification of candicidin in S4 extracts. Analysis of S4-derived extracts. Left panel (A), ultraviolet spectrum extracted at RT 8.3 min (see panel B) from the S4 extract. The absorption maxima match those previously reported for candicidin $D$ [12]. Right panel (B), LC-MS analysis of S4 extract. lon chromatograms extracted for the molecular ion of candicidin D ( $\mathrm{m} / \mathrm{z}$ 1109.6) are shown. (C), MS2 analysis of the extracted ion $\mathrm{m} / \mathrm{z}$ 1109.6. The fragmentation pattern of the antifungal compound from Streptomyces $\$ 4$ perfectly matched the fragmentation of candicidin as reported previously [12]. The ions highlighted in the Haeder et al. study [12] are labelled in a larger font.

Additional file 4: genome sequencing data for Pseudonocardia P1. Summary of the Pseuodonocardia sp. P1 draft genome sequence output obtained by 454 pyrosequencing

Additional file 5: Identification of the nystatin P1 biosynthetic gene cluster. Tiling of Pseudonocardia sp. P1 contigs (GenBank accession ADUJ00000000) to the NPP biosynthetic gene cluster from $P$. autotrophica (GenBank accession EU108007). * The negative value for PP100949 denotes that the contig extends 4517 bp beyond the nystatinlike Pseudonocardia polyene biosynthetic gene cluster. ${ }^{*}$ Negative values indicate that adjacent contigs overlap.

Additional file 6: $\mathbf{M S}^{3}$ data for nystatin P1. The spectrum shows the fragmentation data of the $\mathrm{m} / \mathrm{z} 1088 \rightarrow 326$ ion. The most frequently observed fragmentation corresponds to loss of water: $m / z 308\left(-1 \mathrm{H}_{2} 0\right)$, $m / z 290\left(-2 \mathrm{H}_{2} \mathrm{O}\right), m / z 272\left(-1 \mathrm{H}_{2} \mathrm{O}\right)$. The $m / z 146$ product ion is consistent with a mycosamine sugar after loss of the hexose (mass difference 180).

\section{Abbreviations}

HC: hydrolyzed chitin; HPLC: high-performance liquid chromatography; MS: mannitol plus soya flour; MS/MS: tandem mass spectrometry; NPP: nystatinlike Pseudonocarda polyene; PCR: polymerase chain reaction; PKS: polyketide synthase.

\section{Acknowledgements}

This work was supported by a UEA-funded PhD studentship (JB) and an MRC Milstein award, G0801721 (MIH, RJMG and DY). MIH is a Research Councils UK Fellow. DY also received support from the Yunnan provincial government (20080A001) and the Chinese Academy of Sciences (0902281081). Genome sequencing was carried out at The Genome Analysis Centre under a Capacity and Capability Challenge project with MIH and MJB. We thank Paul Thomas for his assistance with light microscopy, Colin Kay and Lionel Hill for their assistance with LC-MS/MS, Govind Chandra for his advice on sequence analysis, Neil Gow for Candida strains and the Hutchings group members for their help with actinomycete culture and storage.

\section{Author details}

${ }^{1}$ School of Biological Sciences, University of East Anglia, Norwich, Norwich Research Park, NR4 7TJ, UK. ${ }^{2}$ School of Chemistry, University of East Anglia, 
Norwich, Norwich Research Park, NR4 7TJ, UK. ${ }^{3}$ The Genome Analysis Centre, Norwich, Norwich Research Park, NR4 7UH, UK. ${ }^{4}$ Department of Molecular Microbiology, John Innes Centre, Norwich, Norwich Research Park, NR4 7UH, UK. ${ }^{5}$ State Key Laboratory of Genetic Resources, and Evolution, Ecology, Conservation and Environment Center (ECEC), Kunming Institute of Zoology, Chinese Academy of Sciences, Kunming, Yunnan 650223, China. ${ }^{6}$ School of Medicine, Health Policy and Practice, University of East Anglia, Norwich, Norwich Research Park, NR4 7TJ, UK.

\section{Authors' contributions}

JB carried out the bacterial isolation and identification. RFS and SG contributed equally to this work. RFS carried out the genome sequence analysis. SG carried out the chemical isolations and identification. DH and ND sequenced and assembled the genome. MJB, DWY, RJMG and MIH conceived the study, participated in its design and coordination and helped to draft the manuscript. All authors read and approved the final manuscript.

Received: 29 June 2010 Accepted: 26 August 2010

Published: 26 August 2010

\section{References}

1. Kaltenpoth M: Actinobacteria as mutualists: general healthcare for insects? Trends Microbiol 2009, 17:529-535.

2. Schultz TR, Brady SG: Major evolutionary transitions in ant agriculture. Proc Natl Acad Sci USA 2008, 105:5435-5440.

3. Currie CR: A community of ants, fungi, and bacteria: a multilateral approach to studying symbiosis. Annu Rev Microbiol 2001, 55:357-380.

4. Rodrigues A, Bacci M, Mueller UG, Ortiz A, Pagnocca FC: Microfungal 'weeds' in the leafcutter ant symbiosis. Microb Ecol 2008, 56:604-614.

5. Reynolds HT, Currie CR: Pathogenicity of Escovopsis weberi: The parasite of the attine ant-microbe symbiosis directly consumes the ant-cultivated fungus. Mycologia 2004, 96:955-959.

6. Gerardo NM, Mueller UG, Price SL, Currie CR: Exploiting a mutualism: parasite specialization on cultivars within the fungus-growing ant symbiosis. Proc Biol Sci 2004, 271:1791-1798.

7. Currie CR, Scott JA, Summerbell RC, Malloch D: Fungus-growing ants use antibiotic-producing bacteria to control garden parasites. Nature 1999, 398:701-705

8. Little AEF, Murakami T, Mueller UG, Currie CR: Defending against parasites: fungus-growing ants combine specialized behaviours and microbial symbionts to protect their fungus gardens. Biol Lett 2006, 2:12-16.

9. Wang Y, Mueller UG, Clardy J: Antifungal diketopiperazines from symbiotic fungus of fungus growing ant Cyphomyrmex minutus. J Chem Ecol 1999, 25:935-941.

10. Bot ANM, Ortius-Lechner D, Finster K, Maile R, Boomsma JJ: Variable sensitivity of fungi and bacteria to compounds produced by the metapleural glands of leaf-cutting ants. Insectes Sociaux 2002, 49:363-370.

11. Sen R, Ishak HD, Estrada D, Dowd SE, Hong E, Mueller UG: Generalized antifungal activity and 454-screening of Pseudonocardia and Amycolatopsis bacteria in nests of fungus-growing ants. Proc Natl Acad Sci USA 2009, 106:17805-17810.

12. Haeder S, Wirth R, Herz H, Spiteller D: Candicidin-producing Streptomyces support leaf-cutting ants to protect their fungus garden against the pathogenic fungus Escovopsis. Proc Natl Acad Sci USA 2009, 106:4742-4746.

13. Oh DC, Poulsen M, Currie CR, Clardy J: Dentigerumycin: a bacterial mediator of an ant-fungus symbiosis. Nat Chem Biol 2009, 5:391-393.

14. Mueller UG, Dash D, Rabeling C, Rodrigues A: Coevolution between attine ants and actinomycete bacteria: a reevaluation. Evolution 2008, 62:2894-2912.

15. Kim B-G, Lee M-J, Seo J, Hwang Y-B, Lee M-Y, Han K, Sherman DH, Kim E-S: Identification of functionally clustered nystatin-like biosynthetic genes in a rare actinomycetes, Pseudonocardia autotrophica. J Ind Microbiol Biotechnol 2009, 36:1425-1434.

16. Brautaset $T$, Sekurova ON, Sletta H, Ellingsen TE, Strkm AR, Valla $S$, Zotchev SB: Biosynthesis of the polyene antifungal antibiotic nystatin in Streptomyces noursei ATCC 11455:analysis of the gene cluster and deduction of the biosynthetic pathway. Chem Biol 2000, 7:395-403.

17. Brown R, Hazen EL: Present knowledge of nystatin, an antifungal antibiotic. Trans N Y Acad Sci 1957, 19:447-456.
18. Jørgensen H, Fjaervik E, Hakvåg S, Bruheim P, Bredholt H, Klinkenberg G, Ellingsen TE, Zotchev SB: Candicidin biosynthesis gene cluster is widely distributed among Streptomyces spp. isolated from the sediments and the neuston layer of the Trondheim fjord, Norway. Appl Environmental Microbiol 2009, 75:3296-3303.

19. Kroiss J, Kaltenpoth M, Schneider B, Schwinger M-G, Hertweck C, Maddula RK, Strohm E, Svatoš A: Symbiotic streptomycetes provide antibiotic combination prophylaxis for wasp offspring. Nat Chem Biol 2010, 6:261-263.

20. de Kruijff B, Demel RA: Polyene antibiotic-sterol interactions in membranes of Acholeplasma laidlawii cells and lecithin liposomes. 3. Molecular structure of the polyene antibiotic-cholesterol complexes. Biochim Biophys Acta 1974, 339:57-70.

21. Van Leeuwen MR, Golovina EA, Dijksterhuis J: The polyene antimycotics nystatin and filipin disrupt the plasma membrane, whereas natamycin inhibits endocytosis in germinating conidia of Penicillium discolor. J Appl Microbiol 2009, 106:1908-1918.

22. Hsu SC, Lockwood JL: Powdered chitin agar as a selective medium for enumeration of actinomycetes in water and soil. Appl Microbiol 1975, 29:422-426.

23. Kieser T, Bibb MJ, Buttner MJ, Chater KF, Hopwood DA: Practical Streptomyces Genetics. Norwich: John Innes Foundation 2000.

24. Hugenholtz P, Goebel BM, Pace NR: Impact of culture-independent studies on the emerging phylogenetic view of bacterial diversity. J Bacteriol 1998, 180:4765-4774.

25. Lane J: 16S/23 S rRNA sequencing. Nucleic Acid Techniques In Bacterial Systematics NY: John Wiley and Sons 1991, 115-175.

26. Aziz RK, Bartels D, Best AA, Dejongh M, Disz T, Edwards RA, Formsma K, Gerdes S, Glass EM, Kubal M, et al: The RAST Server: rapid annotations using subsystems technology. BMC Genomics 2008, 9:75.

27. Finn RD, Mistry J, Tate J, Coggill P, Heger A, Pollington JE, Gavin OL, Gunasekaran P, Ceric G, Forslund K, et al: The Pfam protein families database. Nucleic Acids Res 2010, 38:D211-D222.

28. Jenke-Kodama H, Dittmann E: Bioinformatic perspectives on NRPS/PKS megasynthases: advances and challenges. Nat Prod Rep 2009, 26:874-883.

29. Kurtz B, Phillipy A, Delcher AL, Smoot M, Shumway M, Antonescu C, Salzberg SL: Versatile and open software for comparing large genomes. Genome Biol 2004, 5:R12.

30. Rutherford K, Parkhill J, Crook J, Horsnell T, Rice P, Rajandream MA, Barrell B: Artemis: sequence visualization and annotation. Bioinformatics 2000, 16:944-945

\section{doi:10.1186/1741-7007-8-109}

Cite this article as: Barke et al:: A mixed community of actinomycetes produce multiple antibiotics for the fungus farming ant Acromyrmex octospinosus. BMC Biology 2010 8:109.

\section{Submit your next manuscript to BioMed Central and take full advantage of:}

- Convenient online submission

- Thorough peer review

- No space constraints or color figure charges

- Immediate publication on acceptance

- Inclusion in PubMed, CAS, Scopus and Google Scholar

- Research which is freely available for redistribution 